\title{
ANP Row Sensitivity
}

\author{
Bill Adams \\ Decision Lens Inc. \\ DeLand FL, USA \\ wjadams@decisionlens.com
}

May 10, 2011

\begin{abstract}
Sensitivity analysis in ANP has several issues surrounding it. In this paper a new theoretical basis for sensitivity analysis is presented which simplifies sensitivity as well as providing a more useful analysis.
\end{abstract}

\section{Introduction}

The goal of sensitivity analysis is to discover how changes in the numerical information in an ANP model effect the scores for the model's alternatives. The numerical data involved could be information directly supplied to the model, such as pairwise data. On the other hand we could also want to analyze sensitivity to calculated data, such as local priorities, or global priorities. These methods do indeed show us certain levels of sensitivity. However, for the vast majority of single level ANP models, they either report useless information (tweaking global priorities is only useful in multi-level models at best), or no sensitivity (a single pairwise comparison has no effect in a well connected ANP model, likewise a single local priority has no effect in a well connected ANP model).

In this paper we construct a new type of sensitivity analysis that gives rise to useful sensitivity in single level ANP models, where other methods have failed. We will use the terminology row sensitivity for this new kind of analysis. We will show that, if we accept certain axioms about preserving ANP structure, row sensitivity as outlined here, is the only kind of calculation we can perform (that is, any other analysis will corrupt the basic structure of the model, rendering the results less meaningful).

We feel obliged to note that, although we speak throughout the paper of single level ANP models, row sensitivity is equally useful in multiple level ANP models. In fact, it serves, in many respects, as a superior replacement to global priorities sensitivity analysis, in that the former preserves the overall structure of the model in a way that the latter can not. 


\subsection{Sensitivity in AHP Trees}

As a way of introducing and motivating our topic and procedure let us review the basic idea and result of standard sensitivity analysis in the case of AHP trees. Although the AHP tree case does not necessarily show us the way we will calculate, it does show us the kind of information we would like to glean from sensitivity.

In typical AHP tree sensitivity, we take the local weights for the collection of criteria under a common parent, and drag up or down a particular criteria's weight. Since we are dealing with a tree, a criteria's local weight and global weight are essentially the same (a simple rescaling is the only change that happens to go from local to global). By changing said local weight (or weights) we get new local priorities for the criteria in question, and resynthesize to get new scores for our alternatives. By dragging a single criteria's priority towards one or towards zero, we get an idea of the influence that criteria has on our alternatives.

Notice, in the process of doing AHP tree sensitivity we need only choose the criteria we wish to analyze, and we are then able to see the impact of that criteria on the alternatives. We would like to be able to do a similar analysis in the ANP case.

\subsection{Prior Existing ANP sensitivity ideas}

We have already briefly mentioned most of the prior existing ANP sensitivity ideas. However we would like to collect them together here, and explain why they are unsuitable analogues of AHP tree sensitivity.

Pairwise comparison sensitivity In this analysis, a particular entry in a pairwise comparison matrix (and it's reciprocal on the other side of the matrix) is changed, new local priorities are calculated, and the alternatives are resynthesized accordingly. In order to do this a with respect to node must be chosen, and two other nodes must be chosen. Simply by virtue of all of these choices, this is not a sufficient analogue of AHP tree sensitivity. In addition, nothing useful is found in such analysis, since one pairwise comparison essentially never has an impact (except in a few degenerate cases).

Local priority sensitivity This sensitivity amounts to changing a single entry in the unscaled supermatrix, recalculating the limit matrix, and resynthesizing to arrive at alternative scores. In order to do this analysis we must choose a with respect to node (the column of the supermatrix) as well as the row (the node's whose priority we are changing).

This method has two shortcomings. First we are not analyzing the sensitivity of a single node but rather of the node with respect to another node. Secondly, in nearly all cases, there is simply no sensitivity to witness (much as in the case of pairwise comparison sensitivity). 
Global priority sensitivity In this analysis, we tweak the global priority of a node (that is, after the limit matrix calculation has already occurred). This analysis proceeds by calculating the limit matrix, deriving global priorities from that limit matrix, then tweaking a node's global priority (and rescaling the others), and then resynthesizing. This is problematic in several ways. First, if the model is a single level all calculations are done at the limit matrix level, and we are tweaking after that point so nothing useful has occurred. Second, even if the model is multiple level, by tweaking the global priority of a node after the limit matrix calculation our sensitivity analysis lies outside of much of the ANP theory, and thus feels somewhat foreign. It does have the advantage of showing the sensitivity of the model to a particular node, but at the cost of only working for multiple level models, and working outside of the context of the majority of ANP theory.

\subsection{Proposed solution}

The problem we have is to get an ANP analogue of AHP tree sensitivity that yields similar results. The proposed solution can be thought of as taking the global priorities approach but moving it before the limit matrix calculation. Or, if one prefers, it can thought of as simultaneously performing local sensitivity analysis on every column.

\subsubsection{Idea}

The basic idea is to change every entry in the scaled supermatrix (and then rescale the rest). We could, in fact do the same thing on the unscaled supermatrix, and the vast majority of the description presented below stays identical (the only difference being one rescales only the rest of the priorities in the given cluster, instead of the entire column).

\subsubsection{Difficulty}

The difficulty we face is determining how much to change each entry in the given row of the supermatrix by. In order to keep the analogy with AHP tree sensitivity, we would like to have a single parameter $p$ that we vary between 0 and 1 (corresponding to the local weight in AHP tree sensitivity). By changing that single parameter we would be changing all of the entries in the given row of the scaled supermatrix (again we could do the same in the unscaled supermatrix, the difference in results is that one tells us how sensitive we are to the node globally as opposed to how sensitive we are to the node when viewed as a part of its parent cluster).

The question becomes, for each value of the parameter $p$, what should we change the entries in the given row of the scaled supermatrix to? There are many choices possible, however we will see that up to continuous change of the 
parameter there is only one choice which will preserve the "ANP structure" of the model $^{1}$.

\section{Supermatrix Row Perturbations which Pre- serve ANP Structure}

The idea behind row sensitivity is to perturb or change each entry in a given row of the scaled supermatrix. Of course, when we perturb a single entry in the supermatrix we must correspondingly change the rest of the entries in that column, so that the column still adds up to one. However, the "main change" in a column is to the entry in the given row, and the changes to the rest of the column are mere consequences of that original entry that is changed. Since we will be changing each entry in a row, we will be changing the rest of the entries so that the columns still add to one (by simply rescaling the rest of the entries in that matrix). In order to precisely describe what preserving ANP structure means, we need a bit of notation.

\subsection{Notation and Definitions}

We will use $W$ for the weighted supermatrix, $W_{i, j}$ for the entry in the $i^{t h}$ row $j^{\text {th }}$ column of the weighted supermatrix. We have already mentioned that we want to use a single parameter $p$ between 0 and 1 to describe the perturbation of our supermatrix. Let us define precisely what we mean now.

Definition 1 (Entry perturbation). Let $W$ be the weighted supermatrix of an ANP model. We say $W^{\prime}$ is a perturbation of $W$ in row $i$ column $j$ if:

- $W^{\prime}$ is stochastic of the same dimensions as $W$

- The columns of $W^{\prime}$ agree with the columns of $W$ except for possibly the $j^{\text {th }}$ column.

- The ratios of the entries in the $j^{\text {th }}$ column of $W^{\prime}$ are the same as those of $W$ except possibly the ratios involving the $i^{\text {th }}$ row.

Note 1. The above definition essentially says we have changed the entry in row $i$ column $j$, and rescaled the remainder of the column so that the column still adds to one.

Definition 2 (Matrix space). Let $M_{r, k}(X)$ be the space of matrices with entries in the space $X$.

Definition 3 (Row perturbation). Fix an ANP model (a single level of it) and let $W$ be its weighted supermatrix (whose dimensions are $n \times n$ ). A family of perturbations of $W$ in the $r^{\text {th }}$ row is a continuous function $f:[0,1] \rightarrow$ $M_{n, n}([0,1])$ with the following properties.

\footnotetext{
${ }^{1}$ This fuzzy terminology will be made precise in the coming pages. The basic idea of preserving "ANP structure" is that we do not change the node connections, and we leave ratios of local priorities as unchanged as possible.
} 
1. $f(p)$ is a stochastic matrix.

2. For some $0<p_{0}<1$ we have $f\left(p_{0}\right)=W$. This $p_{0}$ is called the fixed point of the family.

3. $f(p)$ is the result of a sequence of perturbations of $W$ in row $r$ column $j$ as $j$ ranges from 1 to $n$.

When the family of perturbations is clear, we will write $W(p)$ for $f(p)$, abusing notation in order to gain readability.

Definition 4 (Trivial Column). Fix an ANP model (a single level of it) and let $W$ be its weighted supermatrix (whose dimensions are $n \times n$ ). Also fix a row $1 \leq r \leq n$ to consider a family of row perturbations on. A column $j$ of $W$ is called a trivial column for row perturbations on row $r$ (or simply a trivial column) if either the column is zero, or the column has all zero entries except the $r^{\text {th }}$ entry is 1 . A column is call non-trivial if it is not trivial.

\subsection{Basic properties desired}

There are two basic properties we would like a family of perturbations of the $r^{\text {th }}$ row of the weighted supermatrix to have. They deal with the end points of the family as well as the general flow of the family. We will describe the properties as well as the reason for wanting those properties now.

Let's consider $W(0)$. In considering the AHP tree analogy, the parameter $p$ corresponds to the local weight of our node/criteria. So $W(0)$ should reflect what happens when the $r^{t h}$ node is completely unimportant. In other words it should set all of the local weights for the $r^{t h}$ criteria to zero, i.e. make the $r^{t h}$ row of the supermatrix zero. The only question is what we should do with columns that have the $r^{\text {th }}$ row's entry as a 1 (and thus the rest in that column are zero). By continuity considerations we want those columns to remain unchanged. Thus any trivial column should remain unchanged.

Next let's consider $W(1)$. Again considering the AHP tree analogy, the parameter $p$ being set to one places all importance on the node/criteria in question, and zeros out the rest. So the matrix $W(1)$ should have the $r^{\text {th }}$ row with 1's in any column that had non-zero entries in $W$ (the columns that had the $r^{\text {th }}$ row with a zero means there was no connection there, so we should not change those values), and the rest remain zero.

Lastly in the AHP tree case, as the parameter increases the local priority (and hence global priority) increases. Because of the nature of feedback within an ANP model we cannot guarantee this global priority behavior. However we would like to have, as $p$ increases the local priorities for the $r^{\text {th }}$ criteria to increase (i.e. the values in that row of the weighted supermatrix). In other words the coordinate functions for the $r^{\text {th }}$ row of the family of matrices $W(p)$ are increasing functions. 


\subsection{Maintaining proportionality}

We reach what is perhaps the most important consideration about how a family of perturbations of the weighted supermatrix in a given row should behave. There are, of course, many ways we could perturb the values in a given row, based on the information of a single parameter (we could, for instance set all of the entries in that row to that parameter value). However not many of these choices would preserve the overall ANP structure, and this is what we consider now.

The key idea is that we need to maintain proportionality of elements in the supermatrix throughout our family as much as possible. We cannot keep all of the proportions since that would mean the matrix would never change (since the matrix needs to remain stochastic). In fact motivation comes from looking at the row we are perturbing and our axioms that $W(0)$ should zero out that row and $W(1)$ should place all importance on that row.

First consider the information that $W(0)$ must zero out that row. By continuity this means that as $p \rightarrow 0 W(p)$ should go to $W(0)$. Thus, however we change that row we must make sure that as $p \rightarrow 0$ that row goes to zero. If we force ourselves to maintain proportionality in that row no matter what value $p$ has (at least for $p$ close to zero) we can achieve the desired result. For instance think of $p$ as a scaling factor to multiply the row by. Then as $p \rightarrow 0$ that row does go to zero, and maintains proportionality. So it seems we can hope to have proportionality maintained in the row in question for small values of $p$.

However, considering $W(1)$ shows this is not possible for values of $p$ close to 1 . For, if we maintain proportionality in that row, that row cannot go to 1 (in fact the best it could do is have one entry go to one, and the rest would maintain their proportionality to that one). Since it is not possible to maintain proportionality in that row and have that row go to one, we must look elsewhere for a position to maintain proportionality in. If we force the other rows to maintain their proportionality when $p$ is close to 1 , it turns out to maintain the proportionality of the distance from 1 of the entries in our row (which is a useful proportionality to maintain).

Thus the proportionality we expect to maintain depends on the values of our parameter $p$. Although no formal proof has been yet given that these proportionalities are possible we hope to have shown at least why we cannot have proportionality in the row in question as $p$ goes to 1.

\subsection{Formal definition of the main concept}

We will now collect the various ideas presented above into a single definition for the kind of object we wish to study and use to extend the concept of AHP tree sensitivity to the ANP world.

Definition 5 (Family of row perturbations preserving ANP structure). Fix an ANP model (a single level of it) and let $W$ be its weighted supermatrix (whose dimensions are $n \times n)$. A family of perturbations of $W$ in the $r^{\text {th }}$ row $f:[0,1] \rightarrow$ $M_{n, n}([0,1])$ preserves the ANP structure if: 
1. Trivial columns remain unchanged throughout the family. In other words if the $j^{\text {th }}$ column of $W$ is trivial then the $j^{\text {th }}$ column of $f(p)$ equals the $j^{\text {th }}$ column of $W$ for all $0 \leq p \leq 1$.

2. If $W_{r, i}$ is zero then the $i^{\text {th }}$ column of $f(p)=W(p)$ equals the $i^{\text {th }}$ column of $W$ for all $p$ (that is, if there is no connection from $i$ to $r$ we will not create one ever in the family).

3. If $W_{r, i}$ is non-zero and the $i^{\text {th }}$ column of $W$ is non-trivial, then $W(p)_{r, i}$ is not zero except for $p=0$ (that is the connection from $i$ to $r$ is not broken except when $p=0$ and all influence is removed from node $r$ ).

4. If $p_{0}$ is the parameter for which $W\left(p_{0}\right)=W$ then for $p<p_{0} W(p)$ 's $r^{\text {th }}$ has the same proportionality as $W$ 's $r^{\text {th }}$ row. That is, for $p<p_{0}$ we have

$$
\frac{W_{r, i}}{W_{r, j}}=\frac{W(p)_{r, i}}{W(p)_{r, j}}
$$

where these fractions are defined.

5. For $p>p_{0}$ we have for all $i, i^{\prime} \neq r$ :

$$
\frac{W_{i, j}}{W_{i^{\prime}, j^{\prime}}}=\frac{W(p)_{i, j}}{W(p)_{i^{\prime}, j^{\prime}}}
$$

where these fractions are defined. That is maintain proportionality off the $r^{\text {th }}$ row.

6. We say that the family is increasing if $W(p)_{r, i}$ is an increasing function if $W_{r, i}$ is not zero, and is the constant function zero if $W_{r, i}=0$.

With this we have a definition of a family of row perturbations that preserve ANP structure, and good reasons to accept this as useful definition. However we do not yet know if such families exist.

\subsection{Existence}

In fact such families do exist, as we shall now prove. First we should define our proposed family, and then prove it preserves the ANP structure.

Definition 6. Fix an ANP model (a single level of it) and let $W$ be its weighted supermatrix (whose dimensions are $n \times n)$, and fix $r$ an integer between 1 and n. Pick $0<p_{0}<1$, and define $F_{W, r, p_{0}}:[0,1] \rightarrow M_{n, n}([0,1])$ in the following fashion. Firstly leave trivial columns unchanged throughout the family. Next, if $0 \leq p \leq p_{0}$ define $F_{W, r, p_{0}}(p)$ by scaling the the $r^{\text {th }}$ row by $\frac{p}{p_{0}}$, and renormalizing the columns. If $p_{0} \leq p \leq 1$ define $F_{W, r, p_{0}}(p)$ by leaving alone columns of $W$ for which $W_{r, i}=0$ and scaling all entries in the other columns, except for the entry in the $r^{\text {th }}$ row, by $\frac{1-p}{1-p_{0}}$. 
Note 2. We must be a bit careful as we have defined the above function in two ways for $p=p_{0}$. However using either formula we get the result of $W$ when we plug in $p=p_{0}$ so that the above function is well defined.

Note 3. The above function is a piecewise defined function whose pieces are linear, and they agree at the intersection of the two regions of definition. Thus the above function is continuous.

Theorem 1. Fix an ANP model (a single level of it) and let $W$ be its weighted supermatrix (whose dimensions are $n \times n$ ), fix $r$ an integer between 1 and $n$, and pick $0<p_{0}<1$. Then $F_{W, r, p_{0}}(p)$ is a family of row perturbations preserving the ANP structure.

Proof. It is clear that $F_{W, r, p_{0}}(p)$ satisfies the three conditions for being a family of row perturbations, thus we need only demonstrate that it preserves the ANP structure. However the preservation of ANP structure simply follows from the definitions. In addition it is clear that $F_{W, r, p_{0}}(p)$ is increasing as well.

\subsection{Uniqueness}

Thus we have a family of row perturbations which preserves the ANP structure, which is useful. However, what is surprising is that this family is essentially the only family preserving the ANP structure, up to change of parameter. Let us make this precise.

Theorem 2 (Uniqueness). Fix an ANP model (a single level of it) and let $W$ be its weighted supermatrix (whose dimensions are $n \times n$ ), fix $r$ an integer between 1 and $n$, and pick $0<p_{0}<1$. Let $f(p)$ be a family of row perturbations preserving the ANP structure with $p_{0}$ as the fixed point. Then there exists a continuous map $h:[0,1] \rightarrow[0,1]$ so that

$$
f=F_{W, r, p_{0}} \circ h
$$

Proof. We will define $h(p)$ piecewise, first for $0 \leq p \leq p_{0}$ and then $p_{0} \leq p \leq 1$. Let $0 \leq p \leq p_{0}$. Then $f(p)$ preserves ratios in the $r^{t h}$ row, i.e. the $r^{t h}$ row is a scalar multiple of the $r^{t h}$ row of $W$, let $j$ be a column for which $W_{r, j} \neq 0$. We can calculate that scalar as

$$
\frac{f(p)_{r, j}}{W_{r, j}}
$$

and thus we define

$$
h(p)=p_{0} \cdot \frac{f(p)_{r, j}}{W_{r, j}} .
$$

Since $f$ is continuous its $(r, j)$ entry function is continuous and thus $h$ is continuous. Notice that $h\left(p_{0}\right)=p_{0}$, and that we can determine $F_{W, r, p_{0}} \circ h(p)_{r, j}$ using 
the following sequence of equalities.

$$
\begin{aligned}
F_{W, r, p_{0}} \circ h(p)_{r, j} & =F_{W, r, p_{0}}(h(p))_{r, j} \\
& =F_{W, r, p_{0}}\left(p_{0} \cdot \frac{f(p)_{r, j}}{W_{r, j}}\right) \\
& =W_{r, j} \cdot\left(p_{0} \cdot \frac{f(p)_{r, j}}{W_{r, j}}\right) \cdot \frac{1}{p_{0}} \\
& =f(p)_{r, j}
\end{aligned}
$$

Since $f$ and $F_{W, r, p_{0}}$ preserve the ANP structure and agree in the $(r, j)$ entry, they agree in all entries. Thus for $0 \leq p \leq p_{0}$

$$
f(p)=F_{W, r, p_{0}} \circ h(p) .
$$

Next for $p_{0} \leq p \leq 1$ we note that $f(p)$ preserves the ratios of the rows other than $r$, since $f$ preserves the ANP structure. Let $W_{i, j}$ be a non-zero entry with $i \neq r$. Since $f$ preserves the ratios of rows other than the $r^{t h}$ row, we have a simple scalar multiplication of those rows. We can calculate that scalar as

$$
\frac{f(p)_{i, j}}{W_{i, j}}
$$

and we define $h(p)$ for $p_{0} \leq p \leq 1$ as

$$
h(p)=1-\frac{f(p)_{i, j}}{W_{i, j}}\left(1-p_{0}\right) .
$$

Notice that $h(p)$ as defined above is continuous since $f$ 's entries are continuous and that $h\left(p_{0}\right)=p_{0}$ (thus both definitions agree at their overlap of $p_{0}$, so there is no ambiguity in our definition). Furthermore we can see the following equalities.

$$
\begin{aligned}
F_{W_{r}, p_{0}} \circ h(p)_{i, j} & =F_{W, r, p_{0}}(h(p))_{i, j} \\
& =\frac{1-h(p)}{1-p_{0}} W_{i, j} \\
& =\frac{1-\left(1-\frac{f(p)_{i, j}}{W_{i, j}}\left(1-p_{0}\right)\right)}{1-p_{0}} W_{i, j} \\
& =\frac{\frac{f(p)_{i, j}}{W_{i, j}}\left(1-p_{0}\right)}{1-p_{0}} W_{i, j} \\
& =f(p)_{i, j}
\end{aligned}
$$

Since $f$ and $F_{W, r, p_{0}}$ preserve the ANP structure and agree in the $(i, j)$ entry, they agree in all entries. Thus for $p_{0} \leq p \leq 1$

$$
f(p)=F_{W, r, p_{0}} \circ h(p) .
$$


Thus we have demonstrated $h:[0,1] \rightarrow[0,1]$ which is continuous (since the piecewise parts are continuous and they agree on the overlap) which satisfies

$$
f(p)=F_{W, r, p_{0}} \circ h(p) .
$$

for all $0 \leq p \leq 1$

Remark 1. The previous theorem states that there is only one way to do row sensitivity in way that preserves the ANP structure (up to change of parameter).

\section{$3 \quad$ Example calculations}

So that we may see how these results play out, let us consider a few examples calculated by hand.

\section{$3.1 \quad$ Two node model}

This model contains just two nodes in a single cluster, fully connected. The weighted supermatrix (which is really just the unweighted supermatrix in this case) is

$$
W=\left[\begin{array}{ll}
.2 & \frac{2}{3} \\
.8 & \frac{1}{3}
\end{array}\right]
$$

With this supermatrix we get the normalized priority vector for the alternatives (which we denote as $A$ ):

$$
A=\left[\begin{array}{l}
0 . \overline{45} \\
0 . \overline{54}
\end{array}\right]
$$

We will do row sensitivity on the second row, using parameter values of .1 and .9 (which corresponds to pushing down the priority of the second row for $p=.1$ and pushing it up for $p=.9$ ). For simplicity we will use $p_{0}=.5$.

As a matter of notation we will use $A_{p}$ to denote the new synthesized normalized values of the alternatives when we do row sensitivity with value $p$, and $L_{p}$ for the limit matrix when the parameter is $p$.

$\mathbf{p}=\mathbf{0 . 1}$ : Let us calculate $F_{W, 2,0.5}(0.1)$ first (and then we will need to calculate the limit matrix). Using our formula we will scale row 2 by $.1 / .5=.2$. Thus row two of our new matrix will be .16 and $.2 / 3$. Normalizing our columns we get the first row is .84 and $2.8 / 3$. Thus

$$
F_{W, 2,5}(0.1)=\left[\begin{array}{cc}
.84 & 2.8 / 3 \\
.16 & .2 / 3
\end{array}\right] .
$$

The limit matrix is therefore:

$$
L_{0.1}=\left[\begin{array}{ll}
0.853659 & 0.853659 \\
0.146341 & 0.146341
\end{array}\right]
$$


which gives the new synthesized priorities of

$$
A_{0.1}=\left[\begin{array}{l}
0.853659 \\
0.146341
\end{array}\right]
$$

which has substantially reduced the score of the second alternative from the original values. This is what we would expect by analogy with AHP tree sensitivity. We have decreased the importance of the second alternative prior to calculating the limit matrix, and thus its overall priority has decreased after calculating the limit matrix.

$\mathbf{p}=\mathbf{0 . 9}$ : Again let us calculate $F_{W, 2,0.5}(0.9)$ first and then proceed to the limit matrix. Using the definition we will scale the rows other than 2 (i.e. row one) by $\frac{1-0.9}{1-0.5}=0.2$. Thus the first row becomes .04 and $.4 / 3$. Renormalizing the columns yields the second row as .96 and $2.6 / 3$. Thus

$$
F_{W, 2,0.5}(0.9)=\left[\begin{array}{cc}
.04 & .4 / 3 \\
.96 & 2.6 / 3
\end{array}\right] \text {. }
$$

The limit matrix is therefore:

$$
L_{0.9}=\left[\begin{array}{ll}
.1219512190402955 & .1219512195777367 \\
.8780487809597046 & .8780487804222634
\end{array}\right]
$$

which gives the new synthesized priorities of

$$
A_{0.9}=\left[\begin{array}{l}
.121951 \\
.878049
\end{array}\right]
$$

which has substantially increased the score of the second alternative from the original values. Again this result is as we would expect.

\section{$3.2 \quad$ Four node model}

This is a model with two clusters each of which have two nodes (thus four nodes altogether). There is a single criteria cluster, and the alternatives clusters. In the criteria cluster there are criteria $A$ and $B$. In the alternatives cluster are two nodes, alt 1 and alt2. Everything in the model is fully connected and the weighted supermatrix, and alternative scores are as follows (the order of the nodes being $A, B$, alt 1 , and finally alt2).

$$
\begin{gathered}
W=\left[\begin{array}{llll}
0.375 & 0.20 & 0.175 & 0.10 \\
0.125 & 0.30 & 0.325 & 0.40 \\
0.400 & 0.05 & 0.275 & 0.15 \\
0.100 & 0.45 & 0.225 & 0.35
\end{array}\right] \\
A=\left[\begin{array}{l}
0.388144 \\
0.611856
\end{array}\right]
\end{gathered}
$$

As before we will set $p=0.1$ first, then $p=0.9$, and we will work with criteria B sensitivity (i.e. row 2 ) and $p_{0}=0.5$. 
$\mathbf{p}=\mathbf{0 . 1}$ : First we calculate the new matrix. For $p=0.1$ we scale row 2 by $0.1 / 0.5=0.2$, and then renormalize. We get

$$
F_{W, 2,0.5}(0.1)=\left[\begin{array}{cccc}
.417857 & .268571 & .242407 & .153333 \\
0.025 & 0.06 & 0.065 & 0.08 \\
.445714 & .067143 & .380926 & .536667 \\
.111429 & .604286 & .311667 & 0.349993
\end{array}\right]
$$

The limit matrix result is:

$$
L_{0.1}=\left[\begin{array}{llll}
.2572 & .2572 & .2572 & .2572 \\
.0598 & .0598 & .0598 & .0598 \\
.3248 & .3248 & .3248 & .3248 \\
.3583 & .3583 & .3583 & .3583
\end{array}\right]
$$

This yields the following synthesized priorities for alt 1 and alt2.

$$
A_{0.1}=\left[\begin{array}{l}
.4758 \\
.5242
\end{array}\right]
$$

$\mathbf{p}=\mathbf{0 . 9}$ : Let us calculate the new matrix. Using our formula we will multiply rows 1,3 , and 4 by $\frac{1-0.9}{1-0.5}=0.2$, and then change row 2 to normalize the columns. This gives us

$$
F_{W, 2,0.5}(0.9)=\left[\begin{array}{llll}
.075 & .040 & .035 & .020 \\
.825 & .860 & .875 & .880 \\
.080 & .010 & .055 & .030 \\
.020 & .090 & .045 & .070
\end{array}\right]
$$

the limit matrix is thus

$$
L_{0.9}=\left[\begin{array}{llll}
.039761 & .039761 & .039761 & .039761 \\
.863877 & .863877 & .863877 & .863877 \\
.015210 & .015210 & .015210 & .015210 \\
.085178 & .085178 & .085178 & .085178
\end{array}\right]
$$

and finally the synthesized priorities are

$$
A_{0.9}=\left[\begin{array}{l}
.1515 \\
.8485
\end{array}\right]
$$

\section{Alternate definition of $F_{W, r, p_{0}}(p)$}

The definition given previously for the family of row perturbations $F_{W, r, p_{0}}(p)$ is useful conceptually; however, there is another useful way of defining that family (a different way to write the formula) that only talks about changing the $r^{\text {th }}$ row and rescaling the rest of each column. We describe that formula in terms of the theorem below (stating that the new formulation is the same as our original formulation). 
Theorem 3. Fix an ANP model (a single level of it) and let $W$ be its weighted supermatrix (whose dimensions are $n \times n$ ), and fix $r$ an integer between 1 and $n$. Pick $0<p_{0}<1$. We can define $F_{W, r, p_{0}}:[0,1] \rightarrow M_{n, n}([0,1])$ in the following alternate fashion. First leave trivial columns unchanged throughout the family. Next, for all $0 \leq p \leq 1$ we define $F_{W, r, p_{0}}(p)$ by changing the $r^{\text {th }}$ row and then rescaling the remaining entries in the columns so that the columns continue to add to one. For $0 \leq p \leq p_{0}$ we change the $r^{\text {th }}$ by scaling it by $\frac{p}{p_{0}}$. For $p_{0} \leq p \leq 1$ we change the entries in the $r^{\text {th }}$ row by the following formula

$$
F_{W, r, p_{0}}(p)_{r, j}=1-\alpha\left(1-W_{r, j}\right)
$$

where $\alpha=\frac{1-p}{1-p_{0}}$.

Note 4. The above formulation implies that, for $p_{0} \leq p \leq 1$ we scale the distance from 1 of the entries in the $r^{\text {th }}$ row by $\alpha=\frac{1-p}{1-p_{0}}$.

Proof. Our new definition agrees with the original definition for $0 \leq p \leq p_{0}$, so we need to only consider the other case. Thus let $p_{0} \leq p \leq 1$. We have the formula

$$
F_{W, r, p_{0}}(p)_{r, j}=1-\alpha\left(1-W_{r, j}\right) .
$$

Fix a non-trivial column $j$, we need to show that $F_{W, r, p_{0}}(p)_{i, j}=\frac{1-p}{1-p_{0}} W_{i, j}=$ $\alpha W_{i, j}$ for all $i \neq r$ to prove our definitions coincide.

Let $\beta_{j}$ be the scaling factor we scale the entries of the $j^{\text {th }}$ column by (except for the $r^{t h}$ row). Then $F_{W, r, p_{0}}(p)_{i, j}=\beta W_{i, j}$. Since the $j^{t h}$ column of $F_{W, r, p_{0}}(p)$ adds to one, we get the following sequence of equalities.

$$
\begin{aligned}
1 & =\sum_{i=0}^{n} F_{W, r, p_{0}}(p)_{i, j} \\
& =F_{W, r, p_{0}}(p) r, j+\sum_{i \neq r} F_{W, r, p_{0}}(p)_{i, j} \\
& =1-\alpha\left(1-W_{r, j}\right)+\sum_{i \neq r} \beta_{j} W_{i, j} \\
& =1-\alpha\left(1-W_{r, j}\right)+\beta_{j} \sum_{i \neq r} W_{i, j} \\
& =1-\alpha\left(1-W_{r, j}\right)+\beta_{j}\left(1-W_{r, j}\right)
\end{aligned}
$$

The last equality coming from the factor that the columns of $W$ add to one. We can continue in the following fashion.

$$
\begin{aligned}
1 & =1-\alpha\left(1-W_{r, j}\right)+\beta_{j}\left(1-W_{r, j}\right) \\
\alpha\left(1-W_{r, j}\right) & =\beta_{j}\left(1-W_{r, j}\right) \\
\alpha & =\beta_{j}
\end{aligned}
$$

Thus we are rescaling the entries of the $j^{\text {th }}$ column (except the entry in the $r^{\text {th }}$ row) by $\alpha$, which completes the proof.

Note 5. We have included this formulation, in part, because this is the formulation used in the Super Decisions engine. 


\section{$5 \quad$ Future directions}

The concept of row sensitivity opens up many avenues of analysis not previously available in ANP theory. For instance, there is influence analysis, i.e. which node is most influential to the decision the ANP model is making. Another example would be perspective analysis, which tells how important the alternatives would be if a single node was the only one in the model with weight (however we do not forget the rest of the model in this calculation). Yet another example is marginal analysis, that is, what are the rates of influence of each of the nodes (a derivative calculation). A final example applying row sensitivity would be search for highest rank influence (that is, which node causes rank change first). 